\author{
Boryung Ju \\ Louisiana State University, Baton Rouge, LA, USA
}

Tao Jin

Louisiana State University, Baton Rouge, LA, USA

Brenton Stewart

Louisiana State University, Baton Rouge, LA, USA

\title{
Interdisciplinary Research Collaboration as a Community of Practice (Paper)
}

\begin{abstract}
:
We present an ongoing research project, which takes a qualitative approach using in-depth interview methodology to explore interdisciplinary scientific collaboration activities at a computation and technology research center. The purpose of this research is to understand how a team of scientists and engineers use physical artifacts and social practices to collaborate. We attempt to identify communities of practice that were involved in those scientific collaborations; specific social practices that both scientists and engineers developed and engaged in, as well as those shared artifacts that were used to facilitate interaction among inter- and intra-group members.
\end{abstract}

\section{Résumé:}

\section{Introduction}

The growing complexity and scale of knowledge expansion in scientific and engineering research has accelerated interdisciplinary collaboration (Cummings \& Kiesler, 2005; Chung, et al., 2015; Sonnenwald, 2007). Such interdisciplinary and/or multidisciplinary suggest, "several unrelated academic disciplines are involved in a way that forces them to cross subject boundaries to create new knowledge and theory and solve a common research goal” (Ponte \& Gross, 2011, p. 161). Thus, interdisciplinary collaboration is a collective assemblage of researchers from heterogeneous academic domains. While previous research has revealed differences among academic disciplines (Shrum et al., 2007; Olson et al., 2008; Gardner, 2013), few studies have focused on the social aspect of scientific collaboration. Therefore, we know little about how communities of practice form and contextualize such collaborative activities, which physical artifacts can be used to mediate interactions between collaborators, or how collaborators might use these artifacts to develop and facilitate their social practices. This proposed paper will address this gap.

We present an ongoing research project, which takes a qualitative approach using in-depth interview methodology to explore interdisciplinary scientific collaboration activities at a computation and technology research center. The purpose of this research is to understand how a team of scientists and engineers use physical artifacts and social practices to collaborate. We attempt to identify communities of practice that were involved in those scientific collaborations; 
specific social practices that both scientists and engineers developed and engaged in, as well as those shared artifacts that were used to facilitate interaction among inter- and intra-group members.

\section{Background}

Inter-/multidisciplinary collaborations are likely to face conflicts because fields vary in terms of organization and process (Whitley, 1984), group diversity or collaboration diversity (Walsh \& Maloney, 2007), and social groupings of people with sharing assumptions among them, behavior patterns, and belief about scholarship (Lattuca, 2001). Participants in scientific collaboration often times are required to have relevant knowledge from the related fields (Golde \& Gallagher, 1999), and communication among collaborators may be further complicated by the failure of participants from different backgrounds to recognize the potential for, or even existence of misunderstanding (Walsh \& Maloney, 2007). It is argued that multidisciplinary research efforts advance innovation due to the juxtaposition of ideas, knowledge, and people. Cummings and Kiesler (2007) posit, this type of collaboration requires a coordination crossing both domain and organizational boundaries. Although there are trade-offs between the benefits and risks, these relationships are nevertheless worthwhile because of the numerous advantages articulated by Katz and Martin (1997) such as: sharing, transfer of knowledge and skills, and most importantly the "cross-fertilization of ideas" (p. 14) and intellectual companionship among collaborators.

Science oriented communities of practice are likely comprised of diverse disciplines, institutions, and sometimes community partnerships. These assemblages are rather conspicuous; the challenge lies in identifying the mechanisms or artifacts that facilitate these processes among disparate groups (Wenger, 1998). Boundary objects are inextricably tied to communities of practice because they do the work of translating the phenomena at hand, between members from various domains. A boundary object is "an analytic concept of those scientific objects which both inhabit several intersecting social world... and satisfy the informational requirements of each of them. Boundary objects are objects which are both plastic enough to adapt to local needs and the constraints of the several parties employing them, yet robust enough to maintain a common identity across sites.... They have different meanings in different social worlds but their structure is common enough to more than one world to make them recognizable, a means of translation. The creation and management of boundary objects is a key process in developing and maintaining coherence across intersecting social worlds" (Star and Griesemer, 1989, p. 393). Scientific work in interdisciplinary collaborations revolves around a diverse set of boundary objects (e.g., taxonomies, maps, drawings, databases, models, algorithms). When they are effective, boundary objects 'work' for these collaborations not because the members of the various scientific communities engaged in the effort have the same mental models of these objects, but rather because boundary objects can accommodate a minimal level of shared understanding that provides a common frame of reference and coherence, and enable local meanings, use and adaptation to meet the specialized needs of the various communities.

\section{Research Methods}

Data Collection: Fourteen study participants were recruited from one research institute focusing on computing technology and engineering at a research-intensive university in the southern United States. To select the participants, three criteria were used: (1) holding a faculty status at the research institute; (2) having been involved in interdisciplinary research projects; and (3) being 
willing to participate in our study voluntarily. The initial group of participants were recruited through email and/or phone contacts. Respondents were subsequently asked to recommend additional individuals as likely participants for the study.

In total fourteen individual open-ended, semi-structured interviews were conducted on site in the spring and summer of 2016. Participants were asked to provide retrospective examples of cases or experiences in their interdisciplinary research collaboration. Each interview session lasted from forty-five minutes to one hour. All interview sessions were audio-recorded and then manually transcribed. The transcripts were sent to each study participant for verification purposes. Unlike a structured interview that uses a definite set of the same questions to elicit responses from interviewees, a semi-structured interview is more flexible. It allows the participants to expand on their answers, or the interviewers to adjust the order of the questions and ask additional, improvised questions (Gray, 2009). In order to ensure the accuracy of the transcribed texts from the interview sessions, we used a semi-structured negotiated text approach (Groner et al., 2012; Fontana \& Frey, 2000). It is a process of sharing and editing texts between the interviewees and the interviewers, which enables researchers to develop more reliable interview data by minimizing subjectivity of descriptions by interviewees and enhancing mutual understanding between both parties.

Data Analysis: We conducted an open coding of the transcripts by using QSR NVivo 10 software. The authors independently examined the original (transcribed) texts, searching for identifiable and meaningful chunks, elicited underlying concepts from each of them, and came up with preliminary categories to classify the concepts emerged. These categories were then constantly compared, discussed, reorganized, and collapsed into broader categories when necessary, until they are refined and finalized.

\section{Preliminary Findings}

Several preliminary findings have emerged from our data. First, numerous communities of practices are identified through stories told by participants about their everyday work routines. These communities are mostly project-related, manifesting as both formal and informal. Secondly, we have identified a list of boundary objects that these scientists and engineers used when collaborating with their counterparts. Among them, the most frequently mentioned include drawings and diagrams; literature and documentation; samples; algorithms, equations, and formulas; standards; students; and initial project byproducts, such as code artifacts, proposal drafts, data or data sets, and conceptual or established models. These artifacts are basically consistent with the categories that Star and Griesemer (1989) put forward: repositories, ideal type, coincident boundaries, and standardized forms. Our preliminary results show that the aforementioned artifacts are very loosely defined and often with unstructured forms. Finally, the use of such boundary objects are always implemented through various learning-oriented social practices, such as brainstorming; information requesting and exchanging; as well as negotiation and translation. For example, one participant, a computer scientist who specializes in visual computing, described one of his projects with a group of anthropologists. The project was to help a local law enforcement agency design a tool that would reconstruct unidentified faces, and restore their identity. The participant and his counterparts not only conversed in different languages but were also using different knowledge domains. The team established weekly face-to-face meetings so that everyone understood the project's mission and goals. Drawings, made by the participant, severed as a boundary object that facilitated communication among the disparate constituencies, and played a vital role in knowledge sharing, translating, and standardization processes. Additionally, we argue 
the drawings provided a critical information thread that connected different disciplines, identities, and perspectives in this scientific collaboration.

\section{Reference List:}

Chung, E., Kwon, N., \& Lee, J. (2015). Understanding scientific collaboration in the research life cycle: Bio- and nanoscientists' motivations, information-sharing and communication practices, and barriers to collaboration. Journal of the Association for Information Science and Technology. doi: 10.1002/asi.23520.

Cummings, J. \& Kiesler, S. (2005). Collaborative research across disciplinary and organizational boundaries. Social Studies of Science, 35(5), 703-722.

Cummings, J. \& Kiesler, S. (2008). Who collaborates successfully? Prior experience reduces collaboration barriers in distributed interdisciplinary research. In Proceedings of the 2008 ACM conference on computer supported cooperative work (pp. 437-446). New York, NY, USA.

Fontana, A. \& Frey, J. (2000). The interview from the structured questions to the negotiated texts. Handbook of Qualitative Research, 2nd Ed. Thousand Oaks, CA: Sage Publications. pp. 645-672.

Gardner, S. (2013). Paradigmatic differences, power, and status: A qualitative investigation of faculty in one interdisciplinary research collaboration on sustainability science. Sustainability Science, 8, 241-252.

Golde, C. \& Gallagher, H. (1999). The challenges of conducting interdisciplinary research in traditional doctoral programs. Ecosystems, 2, 281-285.

Gray, D. (2009). Doing Research in the Real World. 2nd Ed. Thousand Oaks, CA: Sage Publications.

Groner, N., Jennings, C., \& Robinson, A. (2012). A negotiated-text method for assessing situation awareness information requirements form emergency responders. 2012 IEEE International Multi-Disciplinary Conference on Cognitive Methods in Situated Awareness and Decision Support, New Orleans, LA.

Katz, J. \& Martin, B. (1997). What is research collaboration? Research Policy, 26, 1-18.

Lattuca, L. (2001). Creating Interdisciplinarity: Interdisciplinary Research and Teaching among College and University Faculty, Vanderbilt University Press, Nashville, TN.

Olson, G.M., Zimmerman, A., \& Bos, N. (2008). Scientific Collaboration on the Internet, MIT Press, Cambridge, MA. 
Ponte, P., Gross, A., Milliman-Richard, Y., \& Lacey, K. (2011). Interdisciplinary teamwork and collaboration: An essential element of a positive practice environment. Annual Review of Nursing Research, 28(1), 159-189.

Shrum, W., Genuth, J., \& Chompalov, I. (eds.) (2007). Structures of Scientific Collaboration, MIT Press, Cambridge, MA.

Star, S. \& Griesemer, J. (1989). "Institutional Ecology, 'Translations' and Boundary Objects: Amateurs and Professionals in Berkeley's Museum of Vertebrate Zoology, 190739". Social Studies of Science, 19 (3), 387-420.

Walsh, J. \& Maloney, N. (2007). Collaboration structure, communication media, and problems in scientific work teams. Journal of Computer-Mediated Communication, 12(2), 712-732.

Wenger, E. (1998). Communities of Practice: Learning, Meaning, and Identity. Cambridge: Cambridge University Press.

Whitley, R. (1984). The fragmented state of management studies: Reasons and consequences. Journal of Management Studies, 21(3), 331-348. 